PROCEEDINGS OF THE

AMERICAN MATHEMATICAL SOCIETY

Volume 138, Number 5, May 2010, Pages 1559-1567

S 0002-9939(09)10016-3

Article electronically published on December 22, 2009

\title{
POLYNOMIALS NON-NEGATIVE ON A STRIP
}

\author{
M. MARSHALL
}

(Communicated by Ted Chinburg)

\begin{abstract}
We prove that if $f(x, y)$ is a polynomial with real coefficients which is non-negative on the strip $[0,1] \times \mathbb{R}$, then $f(x, y)$ has a presentation of the form

$$
f(x, y)=\sum_{i=1}^{k} g_{i}(x, y)^{2}+\sum_{j=1}^{\ell} h_{j}(x, y)^{2} x(1-x),
$$

where the $g_{i}(x, y)$ and $h_{j}(x, y)$ are polynomials with real coefficients.
\end{abstract}

\section{INTRODUCTION}

In 2] Hilbert showed that there are polynomials $f(x, y) \in \mathbb{R}[x, y]$ (necessarily of degree $\geq 6$ ) which are non-negative on all of $\mathbb{R}^{2}$ but are not expressible as a sum of squares in $\mathbb{R}[x, y]$. The best-known example is the polynomial $f(x, y)=$ $1-3 x^{2} y^{2}+x^{4} y^{2}+x^{2} y^{4}[6$. In contrast to this result, we prove:

Theorem 1.1. Suppose $f(x, y) \in \mathbb{R}[x, y]$ is non-negative on the strip $[0,1] \times \mathbb{R}$. Then $f(x, y)$ is expressible as

$$
f(x, y)=\sigma(x, y)+\tau(x, y) x(1-x),
$$

where $\sigma(x, y), \tau(x, y)$ are sums of squares in $\mathbb{R}[x, y]$.

This answers questions in [3] and [10] arising from the solution of the moment problem for cylinders with compact cross-section; see [3] and [16. In [10 the authors claimed to know a proof of the result, but this claim was later withdrawn. Certain weak versions of the result were known already; see [3, [8] and $[9$.

A preordering of a ring $A$ (commutative with 1) is a subset $T$ of $A$ satisfying $T+T \subseteq T, T T \subseteq T$, and $f^{2} \in T$ for all $f \in A$. The unique smallest preordering of $A$ is $\sum A^{2}:=$ the set of all (finite) sums of squares of elements of $A$. The preordering of $A$ generated by finitely many elements $g_{1}, \cdots, g_{s}$ of $A$ consists of all elements of the form $\sum_{i} \sigma_{i} g^{i}, \sigma_{i} \in \sum A^{2}, g^{i}:=g_{1}^{i_{1}} \cdots g_{s}^{i_{s}}, i:=\left(i_{1}, \cdots, i_{s}\right)$ running through the set $\{0,1\}^{s}$.

A finitely generated preordering $T$ of the polynomial ring $\mathbb{R}\left[x_{1}, \cdots, x_{n}\right]$ is said to be saturated if, for all $f \in \mathbb{R}\left[x_{1}, \cdots, x_{n}\right], f \geq 0$ on $K_{T} \Rightarrow f \in T$. Here, $K_{T}:=\left\{a \in \mathbb{R}^{n} \mid \forall g \in T g(a) \geq 0\right\}$. If $g_{1}, \cdots, g_{s}$ are generators of $T$, then $K_{T}$ is the subset of $\mathbb{R}^{n}$ defined by the polynomial inequalities $g_{i} \geq 0, i=1, \cdots, s$.

Received by the editors June 9, 2008, and in revised form, April 26, 2009.

2010 Mathematics Subject Classification. Primary 14P99; Secondary 12D15, 12E05.

Key words and phrases. Positive polynomials, sums of squares, moment problem.

This research was funded in part by an NSERC Discovery Grant.

(C)2009 American Mathematical Society 
By [12, Prop. 6.1], a finitely generated preordering $T$ of $\mathbb{R}\left[x_{1}, \cdots, x_{n}\right]$ cannot be saturated if $\operatorname{dim}\left(K_{T}\right) \geq 3$. By [14, Th. 5.4], the same is true for $\operatorname{dim}\left(K_{T}\right)=2$, if $T$ is stable. The preordering of $\mathbb{R}[x, y]$ consisting of sums of squares is stable, so the result of Hilbert referred to earlier can be seen as a special case of this latter result. Theorem 1.1 asserts that the preordering of $\mathbb{R}[x, y]$ generated by $x(1-x)$ is saturated. The identities $x=x^{2}+(1-x)^{2}$ and $1-x=x(1-x)+(1-x)^{2}$ imply that the preordering generated by $x(1-x)$ coincides with the preordering generated by $x$ and $1-x$. Before the present paper was written, the only example of a finitely generated saturated preordering in the 2-dimensional non-compact case was the rather artificial example given in [15, Rem. 3.14] (the preordering of $\mathbb{R}[x, y]$ generated by $x, 1-x, y$ and $1-x y$ ). It is hoped that the techniques employed in the present paper will yield additional examples of this sort in the future. See [1] and 15] for examples of finitely generated saturated preorderings in the 2-dimensional compact case. See [3, 7], 12] and [13] for 1-dimensional examples.

\section{Preliminary Reductions}

We assume $f \in \mathbb{R}[x, y], f \geq 0$, on the strip $[0,1] \times \mathbb{R}$. We want to show $f$ has a presentation $f=\sigma+\tau x(1-x)$, with $\sigma, \tau$ sums of squares in $\mathbb{R}[x, y]$. By considering the behavior of $f$ as $|y| \rightarrow \infty$, we see that $f$ has even degree $2 d$, as a polynomial in $y$, and that the leading coefficient is $\geq 0$ on [0,1], i.e., $f$ has the form $f(x, y)=\sum_{i=0}^{2 d} a_{i}(x) y^{i}, a_{i}(x) \in \mathbb{R}[x], a_{2 d}(x) \geq 0$ on $[0,1]$. If $d=0$ the result is well-known, e.g., by [3, Th. 2.2] or [4, Prop. 2.7.3], so we assume always that $d \geq 1$.

Lemma 2.1. We may assume $a_{2 d}(x)>0$ on $[0,1]$.

Proof. Factor $a_{2 d}$ as $a_{2 d}=\bar{a} \tilde{a}$, where $\bar{a}, \tilde{a} \in \mathbb{R}[x], \bar{a}$ is $>0$ on $[0,1]$ and $\tilde{a}$ is \pm 1 times a product of linear factors $x-r, r \in[0,1]$. Then

$$
\tilde{a}^{2 d-1} f=\bar{a}(\tilde{a} y)^{2 d}+a_{2 d-1}(\tilde{a} y)^{2 d-1}+\cdots+a_{0} \tilde{a}^{2 d-1} .
$$

Let $g:=\bar{a} y^{2 d}+a_{2 d-1} y^{2 d-1}+\cdots+a_{0} \tilde{a}^{2 d-1}$. Using the fact that $\tilde{a} \geq 0$ on $[0,1]$ and the set of points $(r, s)$ in the strip $[0,1] \times \mathbb{R}$ satisfying $\tilde{a}(r) \neq 0$ is dense in the strip, one sees that $g \geq 0$ on the strip. If we are able to show that $g=\sigma+\tau x(1-x)$, with $\sigma, \tau$ sums of squares in $\mathbb{R}[x, y]$, then $\tilde{a}(x)^{2 d-1} f(x, y)=\sigma(x, \tilde{a}(x) y)+\tau(x, \tilde{a}(x) y) x(1-x)$. Thus we are reduced to showing that if $b(x) f(x, y)$ has a presentation $b(x) f(x, y)=$ $\sigma(x, y)+\tau(x, y) x(1-x)$ for some sums of squares $\sigma(x, y), \tau(x, y)$, where $b(x) \geq 0$ on the interval $[0,1]$ and $b(x)$ is \pm 1 times a product of linear factors $x-r, r \in[0,1]$, then $f(x, y)$ also has such a presentation. The proof is by induction on the degree of $b(x)$. Suppose $x-r$ is a factor of $b(x), 0 \leq r \leq 1$. First suppose $0<r<1$. Then $b(x)=\bar{b}(x)(x-r)^{2}$. Also, $\sigma(x, y)$ and $\tau(x, y)$ vanish at $x=r$, so $\sigma(x, y)=$ $\bar{\sigma}(x, y)(x-r)^{2}, \tau(x, y)=\bar{\tau}(x, y)(x-r)^{2}$, with $\bar{\sigma}(x, y), \bar{\tau}(x, y)$ sums of squares in $\mathbb{R}[x, y]$, and $\bar{b}(x) f(x, y)=\bar{\sigma}(x, y)+\bar{\tau}(x, y) x(1-x)$. If $r=0$, then $b(x)=\bar{b}(x) x$ and $\sigma(x, y)=\bar{\sigma}(x, y) x^{2}$, with $\bar{\sigma}(x, y)$ a sum of squares in $\mathbb{R}[x, y]$, and $\bar{b}(x) f(x, y)=$ $\bar{\sigma}(x, y) x+\tau(x, y)(1-x)$. Using $x=x^{2}+x(1-x)$ and $1-x=(1-x)^{2}+x(1-x)$ this yields $\bar{b}(x) f(x, y)=\sigma^{\prime}(x, y)+\tau^{\prime}(x, y) x(1-x)$, where $\sigma^{\prime}(x, y)$ and $\tau^{\prime}(x, y)$ are sums of squares in $\mathbb{R}[x, y]$. The argument for $r=1$ is basically the same as that for $r=0$.

Lemma 2.2. We may assume $f$ has only finitely many zeros in the strip $[0,1] \times \mathbb{R}$. 
Proof. If $f=g^{2} h$, then, using the fact that the set of points $(a, b)$ in the strip satisfying $g(a, b) \neq 0$ is dense in the strip [4, Prop. 1.1.2], we see that $h \geq 0$ on the strip. So we are reduced to showing the result for $h$. Thus we are reduced to the case where $f$ is square-free. Since $x$ and $1-x$ do not divide $f$ (because they do not divide $a_{2 d}$ ), $f$ has only finitely many zeros on the boundary of the strip. If some irreducible factor $p$ of $f$ has infinitely many zeros in the interior of the strip, then $p$ has a non-singular zero $(a, b)$ in the interior of the strip which is not a zero of any other irreducible factor of $f$ [4, Lem. 9.4.1]. Then $p$ changes sign at $(a, b)$, but each other irreducible factor of $f$ has constant sign in a neighborhood of $(a, b)$, contradicting the fact that $f$ is $\geq 0$ on the strip. It follows that $f$ has only finitely many zeros in the strip.

If $f$ is square-free, then no irreducible factor of $f$ can change sign in the interior of the strip, so each irreducible factor has constant sign on the strip. Replacing $p$ by $-p$ if necessary, for each irreducible factor $p$ we may assume each irreducible factor of $f$ is $\geq 0$ on the strip. In this way we are reduced further to the case where $f$ itself is irreducible. But this does not seem to help us much in the proof.

\section{THE IDEA OF THE PROOF}

Consider the case where the polynomial $f(x, y):=\sum_{i=0}^{2 d} a_{i}(x) y^{i}$ is positive on $[0,1] \times \mathbb{R}$, and $a_{2 d}(x)>0$ on $[0,1]$. The form $F(x, y, z):=\sum_{i=0}^{2 d} a_{i}(x) y^{i} z^{2 d-i}$ is positive for $0 \leq x \leq 1,(y, z) \neq(0,0)$ (since $F(x, y, z)=z^{2 d} f\left(x, \frac{y}{z}\right)$ if $z \neq 0$ ), so it achieves a positive minimum $\epsilon$ on the compact set

$$
[0,1] \times \mathbb{S}^{1}:=\left\{(x, y, z) \mid 0 \leq x \leq 1 \text { and } y^{2}+z^{2}=1\right\} .
$$

Then, on the strip $[0,1] \times \mathbb{R}$,

$$
f(x, y)=F(x, y, 1)=F\left(x, \frac{y}{\sqrt{1+y^{2}}}, \frac{1}{\sqrt{1+y^{2}}}\right)\left(1+y^{2}\right)^{d} \geq \epsilon\left(1+y^{2}\right)^{d} .
$$

Using this, the argument in [3, Th. 5.1] shows that $f(x, y)$ has the required presentation. See [5, Th. 2.2] for additional explanation.

In the general case, one cannot possibly have such an $\epsilon$. The idea is to replace $\epsilon$ by a polynomial $\epsilon(x)$. Specifically, we look for a polynomial $\epsilon(x) \in \mathbb{R}[x]$ such that

$$
f(x, y) \geq \epsilon(x)\left(1+y^{2}\right)^{d}
$$

holds on the strip, $\epsilon(x) \geq 0$ on $[0,1]$, and, $\forall x \in[0,1], \epsilon(x)=0$ iff $f(x, y)=0$ for some $y \in \mathbb{R}$. It is always possible to find such a polynomial $\epsilon(x)$, assuming that $a_{2 d}(x)>0$ on $[0,1]$ and $f(x, y)$ has only finitely many zeros in the strip. Once this is established, one can show that a modified version of the argument in [3, Th. 5.1] carries through, with $\epsilon$ replaced by $\epsilon(x)$. This latter part of the argument is a bit technical: it is necessary to take pains to ensure that the continuous functions considered are analytic at the points where $\epsilon(x)=0$ and to use a refined version of the Weierstrass Approximation Theorem. 


\section{The ADDITIONAL INGREDIENTS}

We establish the additional results that we need in the proof of Theorem 1.1 .

Lemma 4.1. Suppose $f \in \mathbb{R}[x, y]$ is non-negative on a strip of the form $[0, \epsilon) \times \mathbb{R}$, $\epsilon>0, f$ has only finitely many zeros in this strip, and the leading coefficient of $f$ is positive on the interval $[0, \epsilon)$. Then there exists a real constant $C>0$, an even integer $m \geq 0$, and a real number $\delta, 0<\delta \leq \epsilon$, such that $f(x, y) \geq C x^{m}$ holds on the strip $[0, \delta) \times \mathbb{R}$. If $f$ has no real zeros on the $y$-axis, we may take $m=0$.

We denote by $k((x))$ the formal power series field over a field $k$, i.e., the field of fractions of the formal power series ring $k[[x]]$.

Proof. The leading coefficient of $f$ is positive at zero; in particular, it is a unit in $\mathbb{R}[[x]]$. By Puiseux's Theorem, $f$ factors into linear factors in $\mathbb{C}\left(\left(x^{\frac{1}{n}}\right)\right)[y]$, for some $n \geq 1$. Each root has the form $z=\sum_{i=0}^{\infty} a_{i} x^{\frac{i}{n}}, a_{i} \in \mathbb{C}$, and is a complex analytic function of $x^{\frac{1}{n}}$ in a neighborhood of zero [11, Sect. 12.3]. $\bar{z}:=\sum_{i=0}^{\infty} \overline{a_{i}} x^{\frac{i}{n}}$ is another root of $f$, and $\bar{z} \neq z$. (If all the $a_{i}$ were real, then the equation $y=\sum_{i=0}^{\infty} a_{i} x^{\frac{i}{n}}$ would define a real half-branch in the zero set of $f$, for $x$ close to zero, $x>0$, contradicting our assumption that $f$ has only finitely many zeros in the strip $[0, \epsilon) \times \mathbb{R}$.) Write $a_{i}=b_{i}+c_{i} \sqrt{-1}, b_{i}, c_{i} \in \mathbb{R}$, so $z=z_{1}+z_{2} \sqrt{-1}, \bar{z}=z_{1}-z_{2} \sqrt{-1}$, where $z_{1}:=\sum b_{i} x^{\frac{i}{n}}$, $z_{2}=\sum c_{i} x^{\frac{i}{n}}$, so $(y-z)(y-\bar{z})=\left(y-z_{1}\right)^{2}+z_{2}^{2}$. Let $k=\frac{i_{0}}{n}$ be the order of $z_{2}$ at zero, i.e., the least $\frac{i}{n}$ such that $c_{i} \neq 0$. For any real $x>0$ close to zero and any $y \in \mathbb{R}$,

$$
\frac{(y-z)(y-\bar{z})}{x^{2 k}}=\left(\frac{y-z_{1}}{x^{k}}\right)^{2}+\left(\frac{z_{2}}{x^{k}}\right)^{2}
$$

and $\frac{z_{2}}{x^{k}}$ is a real number close to the non-zero real constant $c_{i_{0}}$. This implies there exists a real constant $C>0$ such that $\frac{(y-z)(y-\bar{z})}{x^{2 k}}>C$ for all real $x>0$ sufficiently close to zero and all real $y$. Note: If $f$ has no real zeros on the $y$-axis, then $a_{0}$ is not real; i.e., $c_{0} \neq 0$, so $k=0$. Factoring $f$ as $f(x, y)=a(x) \prod_{i=1}^{d}\left(y-z_{i}\right)\left(y-\overline{z_{i}}\right)$, where $a(x)$ is the leading coefficient, this yields rationals $k_{i} \geq 0$ and real constants $C_{i}>0$ and

$$
\frac{f(x, y)}{\prod_{i=1}^{d} x^{2 k_{i}}}=a(x) \prod_{i=1}^{d} \frac{\left(y-z_{i}\right)\left(y-\overline{z_{i}}\right)}{x^{2 k_{i}}}>a(x) \prod_{i=1}^{d} C_{i}
$$

for all real $y$ and all real $x>0$ sufficiently close to zero. Finally, $f(x, y) \geq C x^{m}$ for any real $x \geq 0$ sufficiently close to zero and any $y \in \mathbb{R}$, where $C$ is $\prod_{i=1}^{d} C_{i}$ times the minimum value of $a(x)$ on $\left[0, \frac{\epsilon}{2}\right]$ and $m$ is the least even integer $\geq 2 \sum_{i=1}^{d} k_{i}$.

Lemma 4.2. Suppose $f(x, y)=\sum_{i=0}^{2 d} a_{i}(x) y^{i}$ is non-negative on the strip $[0,1] \times \mathbb{R}$, $f(x, y)$ has only finitely many zeros in the strip, and $a_{2 d}(x)$ is positive on the interval $[0,1]$. Then there exists a polynomial $\epsilon(x) \in \mathbb{R}[x], \epsilon(x) \geq 0$ on $[0,1]$, such that $f(x, y) \geq \epsilon(x)\left(1+y^{2}\right)^{d}$ holds on the strip and, for each $x \in[0,1], \epsilon(x)=0$ iff there exists $y \in \mathbb{R}$ such that $f(x, y)=0$.

Proof. For each $r \in[0,1]$, by Lemma 4.1] (applied to the new variables $t=x-r$ and $t=r-x$, or just to $t=x-r$, resp., just to $t=r-x$, if $r=0$, resp., if $r=1)$ we have a real constant $C>0$ and an even integer $m \geq 0$ (with $m=0$ if $f$ has no real zeros on the line $x=r)$, such that $f(x, y) \geq C(x-r)^{m}$ holds for all $(x, y)$ in the strip, with $x$ sufficiently close to $r$. By compactness of the interval $[0,1]$, there are finitely many $0 \leq r_{1}<\cdots<r_{k} \leq 1$ and finitely many positive constants $C_{i}$ and 
even integers $m_{i} \geq 0$ (with $m_{i}=0$ if $f$ has no real zeros on the line $x=r_{i}$ ) such that, for each $(x, y)$ in the strip, $f(x, y) \geq C_{i}\left(x-r_{i}\right)^{m_{i}}$, for some $i$. We may assume each $C_{i}$ is $\leq 1$. Then $C_{i}\left(x-r_{i}\right)^{m_{i}} \geq \prod_{j=1}^{k} C_{j}\left(x-r_{j}\right)^{m_{j}}$ for each $x \in[0,1]$ and each $i$. Thus $f(x, y) \geq \epsilon_{1}(x)$ holds on the strip, where $\epsilon_{1}(x):=\prod_{j=1}^{k} C_{j}\left(x-r_{j}\right)^{m_{j}}$.

Since $f(x, y)$ has only finitely many zeros in the strip, there exists a real constant $M>0$ such that $f(x, y)>0$ if $|y| \geq M, 0 \leq x \leq 1$. Arguing with the form $F(x, y, z):=\sum_{i=0}^{2 d} a_{i}(x) y^{i} z^{2 d-i}$ as in Section 3 , but with $\mathbb{S}^{1}=\left\{(y, z) \mid y^{2}+z^{2}=1\right\}$ replaced by the set $\left\{(y, z)\left|y^{2}+z^{2}=1,\right| y|\geq M| z \mid\right\}$, we see there exists a positive constant $C$ such that $f(x, y) \geq C\left(1+y^{2}\right)^{d}$ for all $(x, y)$ in the strip satisfying $|y| \geq M$. If $|y| \leq M$, then $1+y^{2} \leq 1+M^{2}$, and $\frac{f(x, y)}{\left(1+y^{2}\right)^{d}} \geq \frac{f(x, y)}{\left(1+M^{2}\right)^{d}} \geq \frac{\epsilon_{1}(x)}{\left(1+M^{2}\right)^{d}}$. If $|y| \geq M, \frac{f(x, y)}{\left(1+y^{2}\right)^{d}} \geq C \geq \frac{C}{D} \epsilon_{1}(x)$, where $D:=\max \left\{\epsilon_{1}(x) \mid x \in[0,1]\right\}$. So, in any case, $\frac{f(x, y)}{\left(1+y^{2}\right)^{d}} \geq \epsilon(x)$ holds on the strip, where

$$
\epsilon(x):=\min \left\{\frac{1}{\left(1+M^{2}\right)^{d}}, \frac{C}{D}\right\} \epsilon_{1}(x) .
$$

Lemma 4.3. Suppose $f \in \mathbb{R}[x, y]$ and there exists $\epsilon>0$ such that $f$ is non-negative on the strip $(-\epsilon, \epsilon) \times \mathbb{R}$ and the leading coefficient of $f$ is positive on the interval $(-\epsilon, \epsilon)$. Then there exist $g_{1}, g_{2}$ polynomials in $y$ whose coefficients are analytic functions of $x$ defined in a neighborhood of zero, such that $f=g_{1}^{2}+g_{2}^{2}$, for $x$ sufficiently close to zero.

Proof. Let $p$ be an irreducible factor of $f$ in $\mathbb{C}((x))[y]$ which is monic. By Puiseux's Theorem, $p$ factors in $\mathbb{C}\left(\left(x^{\frac{1}{n}}\right)\right)[y]$, where $n$ is the degree of $p$, as $p=\prod_{\omega \in \mu_{n}}\left(y-z_{\omega}\right)$, where $\mu_{n}$ denotes the group of complex $n$-th roots of 1 , and $z_{\omega}=\sum_{i=0}^{\infty} a_{i} \omega^{i} x^{\frac{i}{n}}$ for each $\omega \in \mu_{n}$, where the $a_{i}$ are complex numbers. The $z_{\omega}$ are complex analytic functions of $x^{\frac{1}{n}}$ in a neighborhood of zero [11, Sect. 12.3]. The coefficients of $p$ are elementary symmetric functions of the roots and so are complex analytic functions of $x$ in some neighborhood of zero. Denote by $\bar{p}$ the polynomial in $\mathbb{C}((x))[y]$ obtained from $p$ by conjugating coefficients in the obvious way. $\bar{p}$ is an irreducible factor of $f$. If $\bar{p}=p$, then $z_{1}$ coincides with one of the $\overline{z_{\omega}}:=\sum_{i=0}^{\infty} \overline{a_{i} \omega^{i}} x^{\frac{i}{n}}$. This implies, in turn, that there are (two) real half-branches of $f$ coming from $p 1$ Since $p$ changes sign at any such half-branch, $p$ must appear in $f$ with even multiplicity in this case. Thus $f$ has a factorization of the form $f=a(x) \prod_{i=1}^{k} p_{i} \overline{p_{i}}$, where each $p_{i}$ is irreducible and $a(x)$ is the leading coefficient. Then $f=g \bar{g}$, where $g=\sqrt{a(x)} p_{1} \cdots p_{k}$. Decomposing $g$ as $g=g_{1}+g_{2} \sqrt{-1}, g_{1}, g_{2} \in \mathbb{R}((x))[y]$, this yields $f=g_{1}^{2}+g_{2}^{2}$.

Lemma 4.4. Suppose $f \in \mathbb{R}[x, y]$ is non-negative on the strip $[0,1] \times \mathbb{R}$ and the leading coefficient of $f$ is positive on the interval [0,1]. Then:

(1) For each $r \in(0,1)$, there exist $g_{1}, g_{2}$ polynomials in $y$ with coefficients analytic functions in $x$ in some neighborhood of $r$ such that $f=g_{1}^{2}+g_{2}^{2}$ holds for $x$ sufficiently close to $r$.

\footnotetext{
${ }^{1}$ If $n$ is odd, the real half-branches (one with $x>0$, one with $x<0$ ) are given by $y=\sum a_{i} \tau^{i} x^{\frac{i}{n}}$, where $\tau$ is the unique element of $\mu_{n}$ satisfying $\tau^{2}=\omega$. If $n$ is even, there are two elements, $\tau,-\tau \in \mu_{2 n}$, satisfying $\tau^{2}=(-\tau)^{2}=\omega$. If $\tau,-\tau \in \mu_{n}$, the real half-branches (both with $x>0$ ) are given by $y=\sum a_{i} \tau^{i} x^{\frac{i}{n}}$ and $y=\sum a_{i}(-\tau)^{i} x^{\frac{i}{n}}$. If $\tau,-\tau \notin \mu_{n}$, the real half-branches (both with $x<0)$ are given by $y=\sum a_{i} \tau^{i}(-x)^{\frac{i}{n}}$ and $y=\sum a_{i}(-\tau)^{i}(-x)^{\frac{i}{n}}$.
} 
(2) There exist $g_{i j}, i, j=1,2$, polynomials in $y$ with coefficients analytic functions in $x$ in some neighborhood of 0 such that $f=\sum_{i=1}^{2} g_{i 1}^{2}+\sum_{i=1}^{2} g_{i 2}^{2} x$ holds for $x$ sufficiently close to 0 .

(3) There exist $g_{i j}, i, j=1,2$, polynomials in $y$ with coefficients analytic functions in $x$ in some neighborhood of 1 such that $f=\sum_{i=1}^{2} g_{i 1}^{2}+\sum_{i=1}^{2} g_{i 2}^{2}(1-x)$ holds for $x$ sufficiently close to 1 .

Proof. For (1), apply Lemma 4.3, viewing $f$ as a polynomial in $x-r$ and $y$. For (2), apply Lemma 4.3, viewing $f$ as a polynomial in $\sqrt{x}$ and $y$, to obtain $f=g_{1}^{2}+g_{2}^{2}$ with $g_{i}$ a polynomial in $y$ with coefficients analytic in $\sqrt{x}, i=1,2$. Decomposing each of the coefficients, using $\sum_{k} a_{k} x^{\frac{k}{2}}=\sum_{\ell} a_{2 \ell} x^{\ell}+\sum_{\ell} a_{2 \ell+1} x^{\ell} \sqrt{x}$, yields $g_{i}=g_{i 1}+g_{i 2} \sqrt{x}$, where the $g_{i j}$ are polynomials in $y$ with coefficients analytic functions of $x$ near $x=$ 0 . Expanding $g_{i}^{2}, i=1,2$, then yields $f=\sum_{i=1}^{2} g_{i 1}^{2}+\sum_{i=1}^{2} g_{i 2}^{2} x+2 \sum_{i=1}^{2} g_{i 1} g_{i 2} \sqrt{x}$, so $f=\sum_{i=1}^{2} g_{i 1}^{2}+\sum_{i=1}^{2} g_{i 2}^{2} x$ and $\sum_{i=1}^{2} g_{i 1} g_{i 2}=0$. The proof of (3) is similar to the proof of $(2)$.

Proposition 4.5. Suppose $\phi, \psi:[0,1] \rightarrow \mathbb{R}$ are continuous functions, $\phi(x) \leq \psi(x)$ for all $x \in[0,1]$, and $\phi(x)<\psi(x)$ for all but finitely many $x \in[0,1]$. If $\phi$ and $\psi$ are analytic at each point $a \in[0,1]$ where $\phi(a)=\psi(a)$, then there exists a polynomial $p(x) \in \mathbb{R}[x]$ such that $\phi(x) \leq p(x) \leq \psi(x)$ holds for all $x \in[0,1]$.

Proof 2 Induct on the number of points $a \in[0,1]$ satisfying $\phi(a)=\psi(a)$. If there are no such points, existence of $p(x)$ follows from the Weierstrass Approximation Theorem. Suppose $a \in[0,1]$ is such that $\phi(a)=\psi(a)$. Let $k$ be the vanishing order of $\psi-\phi$ at $a$. If $a \in(0,1)$, then $k$ is even. In this case, $\phi(x)=f(x)+(x-a)^{k} \phi_{1}(x)$, $\psi(x)=f(x)+(x-a)^{k} \psi_{1}(x)$, where $f(x) \in \mathbb{R}[x], \phi_{1}(x), \psi_{1}(x)$ are analytic at $a$, and $\phi_{1}(a)<\psi_{1}(a)$. Extend $\phi_{1}, \psi_{1}$ to continuous functions $\phi_{1}, \psi_{1}:[0,1] \rightarrow \mathbb{R}$ by defining $\phi_{1}(x)=\frac{\phi(x)-f(x)}{(x-a)^{k}}, \psi_{1}(x)=\frac{\psi(x)-f(x)}{(x-a)^{k}}$ for $x \neq a$. Then $\phi_{1}(x) \leq \psi_{1}(x)$ for all $x \in[0,1]$, and, $\forall b \in[0,1], \phi_{1}(b)=\psi_{1}(b)$ iff $\phi(b)=\psi(b)$ and $b \neq a$. By induction we have $p_{1}(x) \in \mathbb{R}[x]$ such that $\phi_{1}(x) \leq p_{1}(x) \leq \psi_{1}(x)$ on $[0,1]$. Take $p(x)=f(x)+(x-a)^{k} p_{1}(x)$. The case where $a=0$ and the case where $a=1$ are dealt with in a similar fashion.

\section{ThE END OF THE PROOF}

Let $f(x, y)=\sum_{i=0}^{2 d} a_{i}(x) y^{i}, d \geq 1$. By Lemmas 2.1 and 2.2 we can assume $a_{2 d}(x)>0$ on the interval and $f(x, y)$ has only finitely many zeros in $[0,1] \times \mathbb{R}$. By Lemma 4.2, there exists a polynomial $\epsilon(x) \in \mathbb{R}[x]$ such that $f(x, y) \geq \epsilon(x)\left(1+y^{2}\right)^{d}$ on the strip, $\epsilon(x) \geq 0$ on $[0,1]$, and $\epsilon(x)=0$ iff $\exists y \in \mathbb{R}$ with $f(x, y)=0$. Let $f_{1}(x, y):=f(x, y)-\epsilon(x)\left(1+y^{2}\right)^{d}$. Then $f_{1}$ is $\geq 0$ on the strip. Replacing $\epsilon(x)$ by $\frac{\epsilon(x)}{N}, N>1$, if necessary, we can assume $f_{1}$ has degree $2 d$ (as a polynomial in $y$ ) and the leading coefficient of $f_{1}$ is positive on $[0,1]$.

By Lemma 4.4 for each $r \in[0,1]$, there exists an open neighborhood $U(r)$ of $r$ in $\mathbb{R}$ such that $f_{1}$ decomposes as

$$
f_{1}=\sum_{j=1}^{2} g_{0 j}(r)^{2}+\sum_{j=1}^{2} g_{1 j}(r)^{2} x+\sum_{j=1}^{2} g_{2 j}(r)^{2}(1-x)
$$

\footnotetext{
${ }^{2}$ Proposition 4.5 is probably well-known. The author only became aware of Proposition 4.5 and its simple proof through reading an unpublished manuscript of V. Powers.
} 
on $U(r) \times \mathbb{R}$, where the $g_{i j}(r)$ are polynomials in $y$ (of degree $\leq d$ ) whose coefficients are analytic functions of $x$, for $x \in U(r) 3$ By compactness of $[0,1]$, finitely many of the $U(r)$ cover $[0,1]$, say $U\left(r_{1}\right), \cdots, U\left(r_{\ell}\right)$ cover $[0,1]$. Choose a continuous partition of unity $1=\nu_{1}+\cdots+\nu_{\ell}$ on $[0,1]$, with $0 \leq \nu_{k} \leq 1$ on $[0,1]$ and $\overline{\operatorname{supp}\left(\nu_{k}\right)} \subseteq U\left(r_{k}\right)$ for $k=1, \cdots, \ell$, having the additional property that, for each root $r$ of $\epsilon(x)$ in $[0,1]$, there is just one $k$ such that $\nu_{k}(x) \neq 0$ close to $r$ (so $\nu_{k}(x)=1$ for $x$ close to $r)$. One way to ensure the last property is to shrink the covering sets $U\left(r_{k}\right)$ ahead of time so that each root $r$ of $\epsilon(x)$ in $[0,1]$ lies in some unique $U\left(r_{k}\right)$. Then $f_{1}$ decomposes as

$$
f_{1}=\sum_{k=1}^{\ell} \nu_{k} f_{1}=\sum_{k=1}^{\ell}\left(\sum_{j=1}^{2} \phi_{0 j k}^{2}+\sum_{j=1}^{2} \phi_{1 j k}^{2} x+\sum_{j=1}^{2} \phi_{2 j k}^{2}(1-x)\right)
$$

on $[0,1] \times \mathbb{R}$, where $\phi_{i j k}$ denotes the polynomial of degree $\leq d$ in $y$ whose coefficients are the functions from $[0,1]$ to $\mathbb{R}$ obtained by extending the corresponding coefficients of $\sqrt{\nu_{k}} g_{i j}\left(r_{k}\right)$ by zero off $U\left(r_{k}\right)$. The coefficients of the $\phi_{i j k}$ are continuous on $[0,1]$ and analytic at each of the roots of $\epsilon(x)$ in $[0,1]$ (since $\nu_{k}$ is constantly 0 or 1 in a neighborhood of each of these roots).

By Proposition 4.5. for each real $N>0$ and each triple $i, j, k$, there exists a polynomial $h_{i j k}$ of degree $\leq d$ in $y$ with coefficients in $\mathbb{R}[x]$ such that, for each coefficient $u$ of $\phi_{i j k}$, the corresponding coefficient $w$ of $h_{i j k}$ satisfies

$$
u(x)-\frac{\epsilon(x)}{N} \leq w(x) \leq u(x)+\frac{\epsilon(x)}{N}, \text { for each } x \in[0,1] .
$$

At this point we proceed as in the proof of [3, Th. 5.1], approximating the coefficients of the $\phi_{i j k}$ closely by polynomials (by taking $N$ sufficiently large), to obtain polynomials $h_{i j k}$ of degree $\leq d$ in $y$ with coefficients in $\mathbb{R}[x]$ such that

$$
\begin{aligned}
& f_{1}(x, y) \\
& =\sum_{k=1}^{\ell}\left(\sum_{j=1}^{2} h_{0 j k}(x, y)^{2}+\sum_{j=1}^{2} h_{1 j k}(x, y)^{2} x+\sum_{j=1}^{2} h_{2 j k}(x, y)^{2}(1-x)\right)+\sum_{i=0}^{2 d} b_{i}(x) y^{i},
\end{aligned}
$$

$b_{i}(x) \in \mathbb{R}[x],\left|b_{i}(x)\right| \leq \frac{2}{5} \epsilon(x)$ on $[0,1], i=0, \cdots, 2 d$. Combining this with $f(x, y)=$ $f_{1}(x, y)+\epsilon(x)\left(1+y^{2}\right)^{d}$ yields $f(x, y)=s_{1}(x, y)+s_{2}(x, y)+s_{3}(x, y)$, where

$$
\begin{aligned}
& s_{1}(x, y):=\sum_{k=1}^{\ell}\left(\sum_{j=1}^{2} h_{0 j k}(x, y)^{2}+\sum_{j=1}^{2} h_{1 j k}(x, y)^{2} x+\sum_{j=1}^{2} h_{2 j k}(x, y)^{2}(1-x)\right) \\
& s_{2}(x, y):=\frac{2}{5} \epsilon(x)\left(2+y+3 y^{2}+y^{3}+3 y^{4}+\cdots+y^{2 d-1}+2 y^{2 d}\right)+\sum_{i=0}^{2 d} b_{i}(x) y^{i}, \\
& s_{3}(x, y):=\epsilon(x)\left[\left(1+y^{2}\right)^{d}-\frac{2}{5}\left(2+y+3 y^{2}+y^{3}+3 y^{4}+\cdots+y^{2 d-1}+2 y^{2 d}\right)\right] .
\end{aligned}
$$

Let $T$ denote the preordering of $\mathbb{R}[x, y]$ generated by $x(1-x)$. As pointed out earlier, $x, 1-x \in T$. Clearly $s_{1}(x, y) \in T$. The argument in [3, Th. 5.1] shows that

\footnotetext{
${ }^{3}$ Applying Lemma4.4 we can choose the $g_{i j}(r)$ so that $g_{2 j}(r)=0, j=1,2$, if $r=0 ; g_{1 j}(r)=0$, $j=1,2$, if $r=1$; and $g_{1 j}(r)=g_{2 j}(r)=0, j=1,2$, if $0<r<1$.
} 
$s_{2}(x, y) \in T$. In more detail, since $\left|b_{i}(x)\right| \leq \frac{2}{5} \epsilon(x)$ on $[0,1], \frac{2}{5} \epsilon(x) \pm b_{i}(x) \in T$, by [3, Th. 2.2] or [4, Prop. 2.7.3], for $i=0, \cdots, 2 d$. This yields

$$
\frac{2}{5} \epsilon(x) y^{i}+b_{i}(x) y^{i} \in T, \text { for } i \text { even. }
$$

For $i$ odd, say $i=2 m+1$, use the identity $y^{2 m+1}=\frac{1}{2} y^{2 m}\left((y+1)^{2}-y^{2}-1\right)$ plus the fact that $\frac{2}{5} \epsilon(x) y^{2 m}(y+1)^{2}+b_{i}(x) y^{2 m}(y+1)^{2}, \frac{2}{5} \epsilon(x) y^{2 m} y^{2}-b_{i}(x) y^{2 m} y^{2}$ and $\frac{2}{5} \epsilon(x) y^{2 m}-b_{i}(x) y^{2 m}$ all belong to $T$ to obtain

$$
\frac{2}{5} \epsilon(x)\left(y^{i+1}+y^{i}+y^{i-1}\right)+b_{i}(x) y^{i} \in T, \text { for } i \text { odd. }
$$

Adding together the various terms of type (5.1) and (5.2), for $i=0, \cdots, 2 d$, we see that $s_{2}(x, y) \in T$. The fact that $s_{3}(x, y)$ belongs to $T$ follows from the identity

$$
\begin{aligned}
& \left(1+y^{2}\right)^{d}-\frac{2}{5}\left(2+y+3 y^{2}+y^{3}+3 y^{4}+\cdots+y^{2 d-1}+2 y^{2 d}\right) \\
& =\left(1+y^{2}\right)^{d}+\frac{1}{5}\left(1+y^{2}+\cdots+y^{2 d-2}\right)(1-y)^{2} \\
& -\frac{8}{5}\left(y^{2}+y^{4}+\cdots+y^{2 d-2}\right)-\left(1+y^{2 d}\right) \\
& =\frac{1}{5}\left(1+y^{2}+\cdots+y^{2 d-2}\right)(1-y)^{2}+\sum_{i=1}^{d-1}\left(\left(\begin{array}{l}
d \\
i
\end{array}\right)-\frac{8}{5}\right) y^{2 i}
\end{aligned}
$$

This means, finally, that $f(x, y)=s_{1}(x, y)+s_{2}(x, y)+s_{3}(x, y) \in T$.

\section{REFERENCES}

[1] J. Cimprič, S. Kuhlmann, M. Marshall, Positivity in power series rings. Adv. Geom., to appear.

[2] D. Hilbert, Über die Darstellung definiter Formen als Summe von Formenquadraten. Math. Ann. 32, 342-350 (1888). MR1510517

[3] S. Kuhlmann, M. Marshall, Positivity, sums of squares and the multidimensional moment problem. Trans. Amer. Math. Soc. 354, 4285-4301 (2002). MR1926876 (2003j:14078)

[4] M. Marshall, Positive polynomials and sums of squares, AMS Surveys and Monographs, 146, Amer. Math. Soc. (2008). MR2383959 (2009a:13044)

[5] M. Marshall, Cylinders with compact cross-section and the strip conjecture. Séminaire de Structures Algébriques Ordonnées, Univ. Paris 6 et 7, June 2008.

[6] T. Motzkin, The arithmetic-geometric inequalities. In: Inequalities, Proc. Symp. WrightPatterson AFB, 1965 (O. Shisha, ed.), Academic Press, 205-224 (1967). MR0223521 $(36: 6569)$

[7] D. Plaumann, Bounded polynomials, sums of squares, and the moment problem. PhD Thesis, Konstanz, 2008.

[8] V. Powers, Positive polynomials and the moment problem for cylinders with compact crosssection. J. Pure and Applied Alg. 188, 217-226 (2004). MR2030815 (2004k:14107)

[9] V. Powers, B. Reznick, Polynomials positive on unbounded rectangles. In: Positive Polynomials in Control, Lecture Notes in Control and Information Sciences, 312 (D. Henrion, A. Garulli, eds.), Springer-Verlag, 151-163 (2005). MR2123522 (2005m:14108)

[10] V. Powers, C. Scheiderer, The moment problem for non-compact semialgebraic sets. Adv. Geom. 1, 71-88 (2001). MR.1823953 (2002c:14086)

[11] G. Sansone, J. Gerretsen, Lectures on the theory of functions of a complex variable, Vol. 2, Wolters-Noordhoff Publishing, Gröningen (1969). MR0259072 (41:3714)

[12] C. Scheiderer, Sums of squares of regular functions on real algebraic varieties. Trans. Amer. Math. Soc. 352, 1039-1069 (2000). MR.1675230(2000j:14090)

[13] C. Scheiderer, Sums of squares on real algebraic curves. Math. Z. 245, 725-760 (2003). MR2020709 (2004k:14103) 
[14] C. Scheiderer, Non-existence of degree bounds for weighted sums of squares representations J. of Complexity 21, 823-844 (2005). MR2182447 (2006k:14117)

[15] C. Scheiderer, Sums of squares on real algebraic surfaces. Manuscripta Math. 119, 395-410 (2006). MR2223624 (2006m:14079)

[16] K. Schmüdgen, The moment problem for closed semi-algebraic sets J. Reine Angew. Math. 558, 225-234 (2003). MR1979186(2004e:47019)

Department of Mathematics and Statistics, University of Saskatchewan, 106 Wiggins Road, Saskatoon, SK, Canada, S7N 5E6

E-mail address: marshall@math.usask.ca 